\title{
Alteraciones tiroideas en la infancia y en la adolescencia. Parte 2: hipotiroidismo
}

Thyroid disorders in childhood and adolescence. Part 2: Hypothyroidism

\author{
Comité Nacional de Endocrinología de la Sociedad Argentina de Pediatría
}

\begin{abstract}
RESUMEN
El hipotiroidismo es la disfunción tiroidea más frecuente, resultante de una disminución de la actividad biológica de las hormonas tiroideas en los tejidos. El objetivo es realizar una revisión y actualización del hipotiroidismo adquirido en la infancia y adolescencia con énfasis en el hipotiroidismo primario. La causa más común es la tiroiditis de Hashimoto o tiroiditis linfocitaria crónica. La característica distintiva es el impacto profundo en el crecimiento esquelético, maduración y desarrollo puberal, con potencial repercusión en la talla adulta. Los signos y síntomas del hipotiroidismo adquirido son similares a los adultos $\mathrm{y}$, en general, no se asocia con compromiso del desarrollo neuromadurativo.

La presunción clínica se confirma con niveles elevados de tirotrofina y disminuidos de tiroxina libre. Las metas del tratamiento incluyen lograr adecuado crecimiento, maduración sexual, desarrolloneuromadurativo y cognitivoóptimo. En la mayoría de los pacientes, el tratamiento de reemplazo revierte los signos y síntomas.

Palabras clave: hipotiroidismo, enfermedad de Hashimoto, bocio, niño, adolescente.
\end{abstract}

\begin{abstract}
Hypothyroidism is the most frequent thyroid dysfunction. It is the consequence of a decrease in the biological activity of thyroid hormones in target tissues. Theaimof this paperistoreview and update acquired hypothyroidism in childhood and adolescence with emphasis on primary hypothyroidism due to its greater frequency. Hashimoto's thyroiditis, also known as chronic lymphocytic thyroiditis, is the most common cause of primary acquired hypothyroidism. The distinctive feature is the profound impact on skeletal growth, maturation, and pubertal development, with potential implications on adult height. Signs and symptoms of acquired hypothyroidism are similar to those reported in adults and are generally not associated with neurodevelopmental impairment. Biochemical
\end{abstract}

Dra. María S. Rodríguez Azrak:

solazrak@gmail.com

Financiamiento:

Ninguno.

Conflicto de intereses:

Ninguno que declarar.

Recibido: 23-12-2019

Aceptado: $12-8-2020$ Dra. María S. Rodríguez Azrak, Dra. Clara Valeri. confirmation of primary hypothyroidism requires the finding of elevated thyrophine and decreased free thyroxine levels. Treatment goals are to achieve normal growth and maturation as well as cognitive development. In most of the patients, replacement treatment reverses symptoms and signs of hypothyroidism and may decrease goiter size.

Key words: hypothyroidism, Hashimoto disease, goiter, child, adolescent.

http:/ / dx.doi.org/10.5546/ aap.2021.S8

Cómo citar: Comité Nacional de Endocrinología. Alteraciones tiroideas en la infancia y en la adolescencia. Parte 2: hipotiroidismo. Arch Argent Pediatr 2021;119(1):S8-S16.

\section{GENERALIDADES}

Durante la infancia, las hormonas tiroideas juegan un rol crítico en el crecimiento, desarrollo y metabolismo, pues modulan la función de la mayoría de los sistemas del organismo. Las consecuencias de sus alteraciones incluyen efectos a nivel cardiovascular, neurológico, cognitivo, gastrointestinal y metabólico.

La disfunción tiroidea en el niño y en el adolescente puede tener múltiples causas, de las cuales la más frecuente es la enfermedad autoinmune. Esta engloba un amplio espectro de disfunciones, que comprenden desde el hipotiroidismo, frecuentemente, asociado a la tiroiditis linfocitaria crónica (TLC) hasta el

Dr. Guillermo Alonso, Dra. Andrea Arcari, Dra. Sonia V. Bengolea, Dra. Elisabeth Boulgourdjian, Dra. Mariana Costanzo, Dra. Silvia D'Amato, Dra. Ana Keselman, Dra. Silvia Martin, Dra. Viviana R. Pipman, 
hipertiroidismo de la enfermedad de Graves (EG), y es mucho más frecuente la TLC (relación $7: 3$ ). ${ }^{1}$

El objetivo de esta publicación es realizar una revisión y actualización de la disfunción tiroidea en la población infantil para su detección y derivación temprana al endocrinólogo. En esta segunda parte, se actualizará sobre hipotiroidismo en la infancia y en la adolescencia.

\section{INTRODUCCIÓN}

El hipotiroidismo primario es la disfunción tiroidea más frecuente, resultante de una disminución de la actividad biológica de las hormonas tiroideas en los tejidos. Es causado por una alteración orgánica o funcional de la glándula tiroides, que provoca déficit en la producción y secreción de hormonas tiroideas: tiroxina (T4), tiroxina libre (T4L), triyodotironina (T3). Otras causas de hipotiroidismo adquirido son menos frecuentes e incluyen al hipotiroidismo hipotálamo-hipofisario, secundario o central ${ }^{1}$ causado por una deficiencia en la estimulación tiroidea por la tirotrofina hipofisaria (thyroid stimulating hormone; TSH, por sus siglas en inglés), a la resistencia a la acción de las hormonas tiroideas (hipotiroidismo periférico) y a la acelerada degradación de estas en los tejidos (hipotiroidismo por consumo). ${ }^{2}$

Según el momento de presentación, puede clasificarse en congénito y adquirido. El hipotiroidismo congénito se presenta desde el nacimiento y tiene una extraordinaria importancia por su potencial repercusión sobre el desarrollo intelectual. En la Argentina, desde la década del 80 (Ley Nacional 23413 sancionada en octubre de 1986 con posteriores modificaciones y reglamentaciones), es obligatoria y universal la realización de la pesquisa neonatal para la detección, entre otras enfermedades, del hipotiroidismo congénito primario. La determinación de TSH permite el diagnóstico precoz y su tratamiento temprano, lo que evita el desarrollo de hipotiroidismo franco con repercusión en el desarrollo mental. ${ }^{3}$

Si el comienzo es más tardío, luego de un período de normalidad, se denomina hipotiroidismo adquirido. En general, no se asocia a retraso mental, con la excepción de aquellos cuadros que se presentan en los primeros dos-tres años de vida. Sin embargo, si no es detectado y tratado durante un tiempo prolongado y, en especial, si es grave, puede generar disfunción cognitiva, alteraciones de la atención, concentración, percepción, función psicomotriz y memoria. ${ }^{4}$ La característica distintiva del hipotiroidismo durante la infancia y la adolescencia es el impacto profundo en el crecimiento esquelético, la maduración y el desarrollo puberal, con potencial repercusión en la talla adulta.., 6

Si bien el objetivo de esta publicación es realizar una revisión y actualización del hipotiroidismo primario adquirido en la infancia y en la adolescencia, es importante tener en cuenta que el hipotiroidismo en los lactantes y en los niños pequeños puede deberse a causas congénitas primarias o centrales no diagnosticadas en el período neonatal:

- Disgenesia tiroidea (tiroides ectópica lingual, hipoplasia) o errores en la biosíntesis de las hormonas tiroideas (disenzimáticos o dishormonogenesis). Aunque, generalmente, se detectan en la pesquisa neonatal, puede ocurrir que, luego de un período de eutiroidismo, se produzca una caída de la producción hormonal y se manifieste el hipotiroidismo durante los primeros tres años de vida. ${ }^{7}$

- Hipotiroidismo central genético. En general, se asocia al déficit de otras hormonas hipofisarias, ya sea debido a mutaciones de genes involucrados en el desarrollo embrionario temprano de la glándula hipofisaria, asociado o no a otras malformaciones (LHX3, LHX4 y HESX1), o a mutaciones de genes en el desarrollo tardío (PROP1, POU1F1). ${ }^{8}$ Mucho menos frecuente es el hipotiroidismo congénito central aislado, con gravedad variable debido a mutaciones del receptor de hormona liberadora de tirotrofina (thyrotropin-releasing hormone; TRH, por sus siglas en inglés), de la subunidad $\beta$-TSH, IGSF1 o TBL1X. ${ }^{9}$ Este tipo de hipotiroidismo no es detectado por los programas de pesquisa basados exclusivamente en la medición de TSH. ${ }^{10}$

\section{HIPOTIROIDISMO ADQUIRIDO Epidemiología y etiología}

El hipotiroidismo es de origen primario en el $99 \%$ de los casos. ${ }^{2}$ La clasificación de su etiología puede verse en la Tabla 1.

La tiroiditis de Hashimoto o tiroiditis linfocitaria crónica (TLC) es la alteración autoinmune más frecuente y la causa más común de hipotiroidismo adquirido tanto en adultos como en niños y adolescentes. ${ }^{11} \mathrm{Su}$ incidencia varía según la etnia, el sexo y la ubicación geográfica; ${ }^{11}$ es del 1,2\% en los niños y 
en los adolescentes en Estados Unidos, y es más frecuente en el sexo femenino (relación F/M: $4-5 / 1) .{ }^{12}$ Suele presentarse con mayor frecuencia entre los 8 y los 15 años de edad, y es excepcional antes de los 5 años. ${ }^{13}$ En el 30-40\% de los casos, hay antecedentes familiares de patología tiroidea. ${ }^{14}$

La TLC es una típica enfermedad autoinmune órgano-específica. La enfermedad tiroidea autoinmune puede manifestarse como TLC y como enfermedad de Graves (EG, publicado en "Parte 1"). En su fisiopatogenia, se han involucrado factores autoinmunes, genéticos y ambientales (Figura 1). Algunos de estos factores son compartidos con la EG. Tanto la EG como la TLC pueden presentarse en una familia; incluso pueden coexistir en la misma glándula. En algunos individuos, la enfermedad tiroidea autoinmune se presenta como EG y, espontáneamente, evoluciona hacia TLC, mientras lo contrario es mucho menos frecuente.

Existe asociación entre TLC y otras enfermedades autoinmunes, como vitiligo, enfermedad celíaca, diabetes mellitus, alopecia areata, y puede también presentarse como parte

TABLA 1. Etiología del hipotiroidismo adquirido

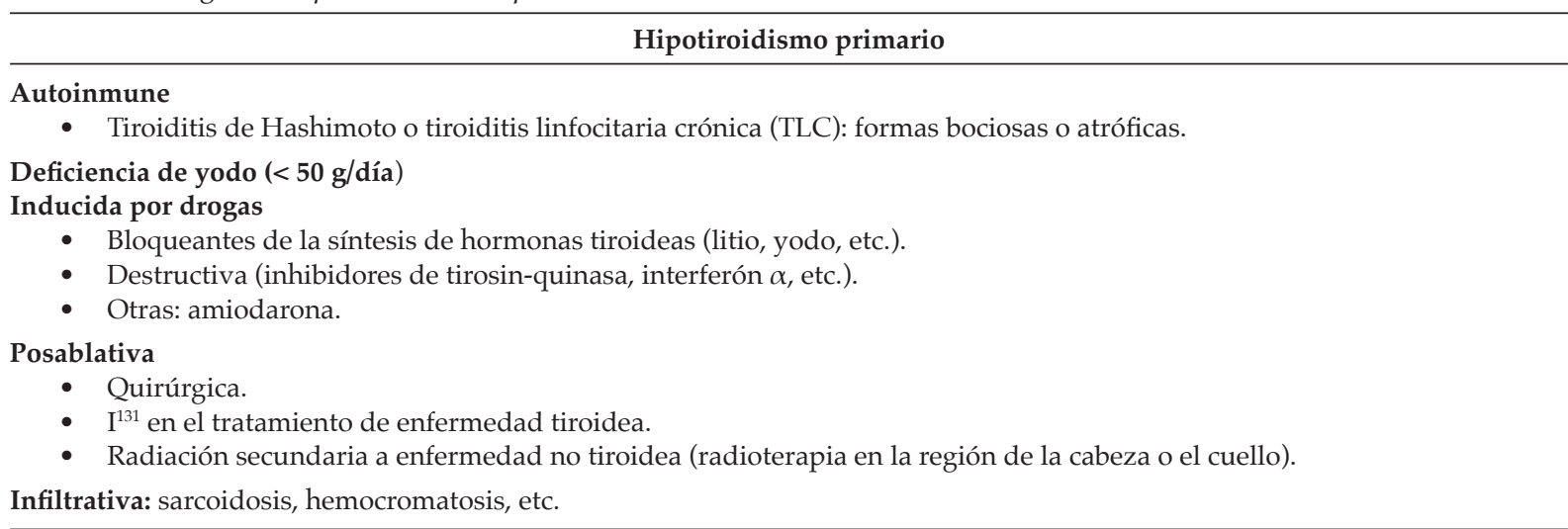

\section{Hipotiroidismo central (secundario o terciario)}

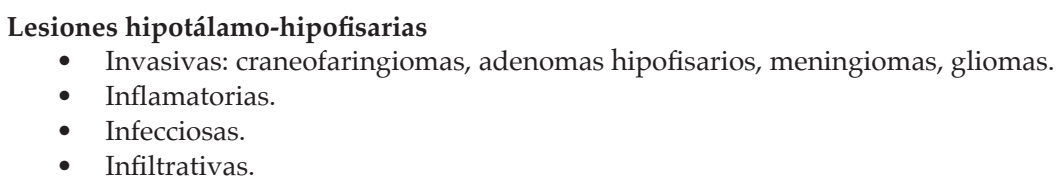

Postratamiento: cirugía, radiación, drogas (somatostatina, dopamina, glucocorticoides, cocaína, carbamazepina, oxcarbazepina, ligando selectivo del receptor de retinoides).

Otras: traumatismo, idiopático.

FiguRA 1. Fisiopatología de la tiroiditis linfocitaria crónica

Fisiopatología multifactorial

Factores inmunológicos

Falla de la tolerancia o vigilancia inmunológica

- Activación de linfocitos T helper CD4

Anticuerpos antitiroideos contra:

Antígenos tiroideos diana

- Tiroperoxidasa (TPG)

- Tiroglobulina $(T g)$

- Receptor de TSH

- Activación de linfocitos T CD 8

$$
\downarrow
$$

Apoptosis celular
Factores genéticos

- Antecedentes familiares en el 30-50\%

- Mayor asociación

Cromosomopatías:

síndrome de Down,

síndrome de Turner

\section{Factores ambientales}

- Bifenilos policlorados

- Infecciones virales, bacterianas

- Yodo en la dieta

- Drogas (INF $\alpha$, amiodarona, litio y antiepilépticos: fenobarbital, carbamazepina, ácido valproico) 
del síndrome poliglandular tipo I y II. La TLC es frecuente en ciertas cromosomopatías, como el síndrome de Down y el síndrome de Turner, que presentan mayor riesgo de enfermedades autoinmunes. ${ }^{15,16}$

Otras de las causas importantes de hipotiroidismo a nivel mundial es la deficiencia de yodo. ${ }^{17} \mathrm{Su}$ incidencia ha disminuido notablemente con el suplemento de yodo en la sal y los alimentos. Cuando el aporte de yodo es menor de $50 \mu \mathrm{g} /$ día, disminuye el contenido de yodo en la glándula tiroides, y se produce una aceleración de todas las etapas del metabolismo intratiroideo del yodo, con bajo nivel de yodación de la tiroglobulina y una reducción de la síntesis de yodotironinas con el siguiente patrón hormonal: T4 baja, TSH elevada y T3 normal o elevada. La estimulación crónica de TSH da lugar al bocio.

Con respecto a los fármacos que pueden alterar los niveles de hormonas tiroideas, la mayoría actúan modificando su unión a las proteínas transportadoras sin alterar los niveles de TSH, habitualmente, después de un período de adaptación. ${ }^{18}$ Sin embargo, hay un grupo de drogas que pueden provocar tiroiditis inducida por fármacos. Se puede manifestar clínicamente como hipertiroidismo o hipotiroidismo y se encuentra vinculada a drogas que pueden actuar por distintos mecanismos, como la sobrecarga yodada (ej.: amiodarona), la expresión de antígenos tiroideos que desencadenan una repuesta autoinmune (ej.: interferón $\alpha$ ) o la depleción de selenoproteínas (valproato) ${ }^{7}$

El hipotiroidismo posterior a la tiroidectomía por hipertiroidismo o cáncer de tiroides es raro en niños. La extirpación involuntaria de tejido tiroideo ectópico durante la resección de un quiste tirogloso puede también ocasionar hipotiroidismo. Por este motivo, antes de la intervención quirúrgica del quiste, se debe realizar una ecografía o un centellograma para la adecuada identificación de la ubicación del tejido tiroideo. ${ }^{19}$

La radioterapia externa secundaria a la enfermedad no tiroidea, como en el caso de tumores de la cabeza y el cuello, enfermedad de Hodgkin o pretrasplante de médula ósea (dosis mayores de $10 \mathrm{~Gy}){ }^{20}$ y el yodo radioactivo $\left(I^{131}\right)$ como tratamiento del hipertiroidismo pueden ocasionar hipotiroidismo. Otras causas más raras son la infiltración del tejido tiroideo (leucemia, histiocitosis, sarcoidosis y hemocromatosis) y los depósitos de cristales de cistina (cistinosis).

El hipotiroidismo central adquirido está típicamente asociado con otras deficiencias hormonales hipofisarias; rara vez, es la primera manifestación de la enfermedad hipotálamohipofisaria. Los antecedentes personales, como radioterapia, consumo de drogas, cirugías o patología del sistema nervioso central, son relevantes y, sumados a la presencia de alteraciones visuales y cefaleas, orientan al diagnóstico.

\section{Manifestaciones clínicas}

El cuadro clínico puede ser muy variable al momento del diagnóstico, ya que los signos y síntomas dependen de la gravedad y de la duración del hipotiroidismo. Más allá de los efectos en el crecimiento, en la maduración y en la pubertad, los signos y síntomas del hipotiroidismo en los niños y en los adolescentes son similares a los de los adultos. Es frecuente la presencia de síntomas leves, inespecíficos y no patognomónicos. La aparición de un síntoma no habitual puede orientar al diagnóstico de hipotiroidismo y, en ese caso, revertirá con el tratamiento. ${ }^{4}$

Las manifestaciones clínicas del hipotiroidismo abarcan numerosos órganos y sistemas (Tabla 2). Entre los signos y síntomas que más se destacan, se encuentran el retardo de crecimiento (que se asocia a retraso en la maduración esquelética), el cansancio o sensación de falta de energía, mal rendimiento escolar, constipación, sequedad de la piel, tendencia al frío y retraso en la dentición. El hipotiroidismo grave puede presentarse con un cuadro que incluye

\section{TABla 2. Signos y sintomas de hipotiroidismo}

- Bocio.

- Fatiga, letargia, somnolencia, depresión.

- Piel seca, fría, gruesa con palidez.

- Pelo y uñas quebradizas.

- Constipación.

- Retraso de crecimiento.

- Retraso puberal.

- Pseudopubertad precoz.

- Retraso de la edad ósea.

- Retraso en la erupción dentaria.

- Retraso en pautas psicomotoras.

- Bradipsiquia.

- Facies abotagada.

- Anorexia.

- Intolerancia al frío.

- Trastornos menstruales.

- Mixedema (edema de manos y pies).

- Hipotonía, hiporreflexia.

- Miopatía e hipertrofia muscular.

- Calambres.

- Bradicardia, hipotensión.

- Derrame pericárdico. 
facies abotagada, cabello ralo y quebradizo o incluso pérdida de este, hipotermia, bradicardia, bradipsiquia, somnolencia, hipotensión sistólica con aumento de la tensión arterial diastólica, retraso en la relajación de los reflejos tendinosos profundos, mixedema, debilidad muscular y pseudohipertrofia. Dejado a su libre evolución, puede ocasionar coma mixedematoso, derrame pleural y pericárdico, hipoglucemia e hiponatremia., ${ }^{9} 11$

El hipotiroidismo adquirido, en general, no se asocia con compromiso del desarrollo neuromadurativo. Sin embargo, como se mencionó previamente, este puede verse comprometido cuando el hipotiroidismo se presenta en los primeros años de vida.

El hipotiroidismo grave ocasiona una abrupta desaceleración en la ganancia de talla coincidente con el aumento relativo de peso, que es secundario al mixedema y no al aumento del tejido adiposo. Muchas veces, se evalúa la función tiroidea en niños con obesidad exógena; sin embargo, es importante destacar que estos últimos no presentan compromiso en la velocidad de crecimiento ni retraso en la maduración esquelética, como sucede en el hipotiroidismo. ${ }^{7}$

Frecuentemente, el hipotiroidismo se asocia a retraso puberal y, en las adolescentes, a alteraciones menstruales. ${ }^{9}$ No obstante, algunas niñas presentan telarca temprana, a veces, incluso menarca, asociada a galactorrea, ausencia de vello pubiano y ovarios de aspecto multiquístico, conocido como síndrome de Van WykGrumbach. ${ }^{21}$ En los varones, puede encontrarse macroorquidismo. ${ }^{22}$ En raros casos, menos del $10 \%$ de los pacientes puede presentarse con una fase inicial de tirotoxicosis, que dura 1-2 meses, llamada hashitoxicosis (producida por la liberación de hormona preformada), que, finalmente, evoluciona al hipotiroidismo. ${ }^{13}$

Ante la presencia de signos y síntomas de hipotiroidismo, debe determinarse el tamaño glandular. Con este fin, es de utilidad comparar los lóbulos tiroideos con la falange distal del pulgar del niño, llamado "calibración individual", y, si son de mayor tamaño, se está en presencia de bocio. ${ }^{7}$ Este es un signo característico de la TLC y es simétrico, difuso y firme ante la palpación. ${ }^{12}$ La TLC puede, ocasionalmente, presentarse sin bocio, que se denomina tiroiditis atrófica, la cual es más frecuente en los niños que en los adultos. ${ }^{23}$ Si no lograra palparse la glándula tiroidea en su localización habitual, deberán examinarse cuidadosamente las fauces y la línea media del cuello en búsqueda de localización tiroidea ectópica ${ }^{7}$ (ej.: tiroides lingual ubicada en la base de la lengua).

\section{DIAGNÓSTICO}

El diagnóstico se basa en la evaluación conjunta de las manifestaciones clínicas, los estudios bioquímicos y las imágenes complementarias. La evaluación de la función tiroidea incluye la medición de las hormonas: TSH, T4, T4L y T3, y de los anticuerpos antitiroideos: antitiroperoxidasa y antitiroglobulina. Es muy importante tener en cuenta que los valores de referencia para las determinaciones varían según la edad y el método bioquímico.

En general, la TSH es el parámetro que mejor refleja la función tiroidea. La presunción clínica de hipotiroidismo primario se confirma con niveles elevados de TSH y disminuidos de T4L (Figura 2). Los valores de TSH de entre 5 y

Tirotrofina (TSH)-tiroxina libre (T4L)

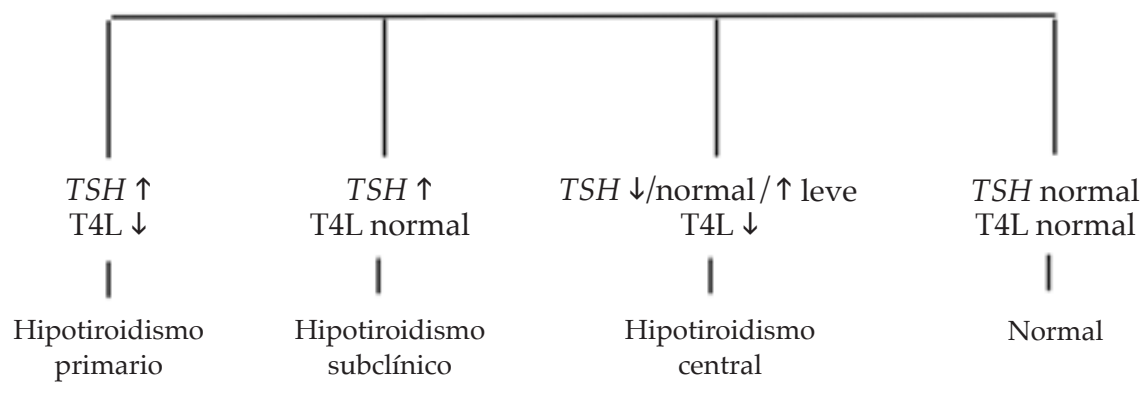

Adaptado de Ladenson PW. Diagnosis of Hypothyroidism. In Braverman LE, Cooper DS (ed.). Werner \& Ingbar`s: The thyroid: a fundamental and clinical text. 10th ed. Philadelphia: Lippincott Williams \& Wilkins; 2013:609. 
$10 \mathrm{mU} / 1$ deberían ser repetidos antes de iniciar el tratamiento, pues, en el $70 \%$ de los casos, serán normales en la siguiente determinación. ${ }^{24}$ Las alteraciones del sueño, el estrés y el calor ${ }^{13}$ pueden modificar los valores de TSH.

Una vez confirmado el diagnóstico de hipotiroidismo primario, el próximo paso es determinar su etiología. En los lactantes o niños menores de 3 años y / o ante la presencia de masa o tumor en la línea media, debe realizarse un centellograma tiroideo con tecnecio para descartar una causa congénita que no haya sido detectada en la pesquisa neonatal.

En los niños y en los adolescentes, deben medirse los autoanticuerpos tiroideos. En caso de niveles elevados de antitiroperoxidasa y antitiroglobulina, se confirma el origen autoinmune: TLC. Esta puede presentarse con o $\sin$ bocio (tiroiditis atrófica autoinmune).

En el hipotiroidismo central, los niveles de TSH pueden ser bajos, normales o estar levemente elevados, pero siempre son inapropiados para los niveles de T4L (Figura 2). Ante la sospecha de hipotiroidismo central, se debe evaluar la existencia de antecedentes personales, como radioterapia, consumo de drogas, cirugías o patología del sistema nervioso central. Los niveles séricos disminuidos de T4 o T4L confirmarán el diagnóstico. Actualmente, no se considera necesaria la realización de un test de estímulo (test de TRH) para su confirmación. ${ }^{23}$ El diagnóstico de hipotiroidismo central obliga a una cuidadosa evaluación del resto de la función hipotálamo-hipofisaria. ${ }^{10,25}$

La ecografía tiroidea es de gran utilidad ante la sospecha de nódulo tiroideo. Este estudio no sería necesario para el diagnóstico de TLC, pero, de realizarse, se puede observar un aumento del volumen glandular, hipoecogenicidad de distribución irregular, alteración difusa del patrón estructural y cambios en la vascularización glandular. ${ }^{26}$

Otros estudios de imágenes que pueden aportar a la evaluación diagnóstica son los siguientes:

- Radiografía de mano y muñeca izquierda (edad ósea), que es útil en la evaluación del retraso de crecimiento. La magnitud del déficit de talla va a ser proporcional a la duración del hipotiroidismo. Se puede estimar el inicio del cuadro clínico calculando la diferencia entre la edad ósea y la edad cronológica.

- Resonancia del sistema nervioso central en el hipotiroidismo central adquirido asociado al déficit de otras trofinas hipofisarias.
Consideraciones especiales en la evaluación de los pacientes con perfil tiroideo alterado, en las cuales se requiere la derivación endocrinológica para determinar la conducta apropiada

- El hipotiroidismo subclínico o hipertirotropinemia, definido por el aumento de los niveles de TSH por encima de los valores de referencia, con valores normales de T4 y T4L. Este cuadro es poco frecuente en niños, del 1,7\%..$^{27} \mathrm{En}$ los pacientes pediátricos, en ausencia de autoanticuerpos, se considera que presenta bajo riesgo de progresar a hipotiroidismo y que no se asocia con la afectación de la función cognitiva ni con la alteración del crecimiento lineal. ${ }^{28,29}$ Dicho patrón bioquímico se encuentra más frecuentemente en niños obesos, en el 10-23\%, en los que se normaliza luego del descenso de peso, por lo que se considera una consecuencia de la obesidad y no su causa. ${ }^{29} \mathrm{El}$ hipotiroidismo subclínico de larga data puede asociarse con hipertensión arterial y alteraciones en el perfil metabólico proaterogénicas, en especial, si se presenta en pacientes obesos. ${ }^{27,28} \mathrm{El}$ factor pronóstico más importante en la evaluación del hipotiroidismo subclínico es la etiología. En pacientes sin enfermedad tiroidea subyacente, la evolución natural se caracteriza por la normalización de los estudios en la gran mayoría de los $\operatorname{casos}^{27}$ (Figura 2).

- Los pacientes con retraso de crecimiento intrauterino (RCIU) sin crecimiento compensatorio pueden presentar valores de TSH moderadamente elevados. ${ }^{30,31}$ En una cohorte argentina de 58 niños con RCIU, el $53 \%$ presentó valores de TSH elevados, en un rango de 6,5 a $14,6 \mu \mathrm{U} / 1$. Los pacientes con TSH elevada que fueron tratados con levotiroxina, si bien normalizaron los valores de $T S H$, no presentaron modificaciones en los niveles de $\mathrm{T} 4 \mathrm{~L}$, en los percentiles de talla ni en el índice de masa corporal al año de tratamiento. Los autores proponen que, si bien las alteraciones del perfil tiroideo son frecuentes en esta población, la ausencia de impacto clínico sugiere que se trataría de una alteración en la modulación de TSH, no una patología en sí misma. ${ }^{32}$

- Los pacientes con síndrome de Down presentan mayor riesgo de enfermedad tiroidea autoinmune (TLC y EG), de hipotiroidismo subclínico o hipertirotropinemia y de hipotiroidismo congénito. ${ }^{16,33}$ Debido a esto, 
la recomendación actual es que, además de la pesquisa neonatal, se debe controlar la función tiroidea a los 6 meses de edad y luego en forma anual. ${ }^{16}$ La enfermedad tiroidea autoinmune en los pacientes con síndrome de Down se suele presentar a edades más tempranas, con menor preponderancia femenina, menor frecuencia de antecedentes familiares y mayor riesgo de asociación con otras patologías autoinmunes. ${ }^{34}$ Algunos autores han reportado bocio con menor frecuencia en pacientes con síndrome de Down. ${ }^{35}$ La prevalencia de hipotiroidismo subclínico es del 7-40 \%, y los niveles más altos de TSH se asocian con mayor riesgo de evolución a hipotiroidismo franco. ${ }^{36}$ Cuando las alteraciones de la función tiroidea son sutiles, los niveles suelen permanecer estables a lo largo del seguimiento y no hay evidencia franca de beneficio con la intervención. . $^{36,37,38}$

- Las situaciones de enfermedad aguda o desnutrición grave producen alteraciones en el patrón bioquímico de las hormonas tiroideas, conocidas como sindrome de enfermedad no tiroidea o sindrome de T3 baja. El hallazgo más frecuente es un nivel sérico bajo de T3 asociado a un incremento en el nivel de T3 reversa y, en casos graves, a niveles bajos de T4 total y libre. Estas alteraciones no se asocian a una elevación concomitante en el nivel de TSH, que puede incluso descender por inhibición del eje hipotálamo-hipofisotiroideo. Se considera, en principio, una respuesta adaptativa a la situación de enfermedad aguda, pero existe controversia sobre si representa un verdadero estado de hipotiroidismo tisular y si requiere tratamiento. Se necesitan estudios controlados para definir estas áreas de incertidumbre. ${ }^{39}$

Existen situaciones, como la hipertirotropinemia, el bocio eutiroideo, TLC eutiroideas, en las que el diagnóstico puede no ser claro, y se requiere control y seguimiento por el especialista para definir una conducta y seguimiento acorde a cada paciente.

\section{TRATAMIENTO}

Las metas del tratamiento durante la infancia y la adolescencia incluyen lograr un adecuado crecimiento y maduración sexual, y un desarrollo neuromadurativo y cognitivo óptimos. E1 tratamiento tardío provoca un impacto negativo en la talla final, especialmente, en pacientes puberales o cercanos al inicio de la pubertad al momento del diagnóstico. ${ }^{5,6}$
En la mayoría de los pacientes, el tratamiento de reemplazo con hormona tiroidea (levotiroxina) revierte los signos y síntomas del hipotiroidismo, y suele disminuir el tamaño del bocio. ${ }^{17} \mathrm{El}$ tratamiento consiste en una dosis única diaria, que debe ser administrada preferentemente en ayunas.

Los suplementos de calcio y hierro, así como algunos alimentos, pueden afectar su absorción, al igual que la presencia de síndromes malabsortivos, como la enfermedad celíaca. ${ }^{9}$ Fármacos como fenitoína, carbamazepina o rifampicina pueden interferir con el metabolismo de hormonas tiroideas y requerir el ajuste de dosis.

La dosis varía según la edad, el peso y la gravedad del hipotiroidismo. En la Tabla 3, se muestra una escala de dosis para el reemplazo completo de la función tiroidea según la edad. ${ }^{40}$ En general, en el hipotiroidismo central, se requieren dosis más bajas. ${ }^{17}$ Es muy importante realizar dosajes hormonales de control a las 6-8 semanas de haberse iniciado el tratamiento, ya que el principal riesgo es el hipertiroidismo iatrogénico. Luego se continúa el seguimiento con monitoreo clínico y estudios de laboratorio cada 4-6 meses. ${ }^{17}$ En el hipotiroidismo primario, el objetivo es mantener niveles de TSH dentro del rango de referencia. ${ }^{9}$

En casos de hipotiroidismo adquirido grave, algunos autores recomiendan iniciar el tratamiento en bajas dosis con un incremento progresivo para evitar eventos adversos, como cefalea, insomnio, hiperactividad y/o déficit de atención. ${ }^{11}$ Se debe informar al paciente y a su familia sobre la importancia del cumplimiento del tratamiento y la necesidad de continuar los controles endocrinológicos.

Si bien existe, en algunos casos, la posibilidad de reversión, la decisión de suspender la medicación y reevaluar la necesidad de tratamiento deberá estar avalada por un endocrinólogo. Se debe advertir a las adolescentes que, ante un embarazo, deben continuar con el tratamiento y realizar una pronta consulta endocrinológica para la adecuación de las dosis.

TABLA3. Dosis de levotiroxina (LT4) acorde a la edad yel peso ${ }^{22}$

\begin{tabular}{lc}
\hline Edad & Dosis $(\mu \mathrm{g} / \mathbf{k g} /$ día $)$ \\
\hline $3-10$ años & $4-6$ \\
$10-16$ años & $2-4$ \\
$\geq 17$ años & 1,6 \\
\hline
\end{tabular}


El hipotiroidismo en la edad pediátrica tiene un gran impacto en diferentes aspectos de la salud en la niñez y en la adolescencia, como el desarrollo neuromadurativo-cognitivo, el crecimiento y el desarrollo puberal. Ante la sospecha clínica de enfermedad tiroidea o frente al hallazgo de resultados fuera del rango de normalidad, se sugiere la pronta evaluación.

\section{CONCLUSIÓN}

La enfermedad tiroidea en la niñez y en la adolescencia es frecuente y puede presentarse con sintomatología muy variada. El pediatra debe estar alerta ante la aparición de síntomas asociados tanto a hipotiroidismo como a hipertiroidismo y ante la presencia de bocio. Se deberá interrogar acerca de los antecedentes personales y/o familiares, y realizar una derivación oportuna al endocrinólogo infantil para favorecer un tratamiento temprano y adecuado, fundamental para garantizar un óptimo crecimiento, metabolismo y desarrollo del niño y del adolescente.

\section{REFERENCIAS}

1. Braverman LE, Cooper DS. Introduction to Hypothyroidism. In Braverman LE, Cooper DS (eds.). Werner EIngbar's: The thyroid: a fundamental and clinical text. $10^{\text {th }}$ ed. Philadelphia: Lippincott Williams \& Wilkins; 2013.Págs.523-5.

2. Huang S. Hypothyroidism. In: Lifshitz F (ed.). Pediatric Endocrinology. 5th ed. New York: Informa Healthcare USA, Inc; 2007; 405-11.

3. Rastogi MV, LaFranchi SH. Congenital hypothyroidism. Orphanet J Rare Dis. 2010; 5:17.

4. Schuff KG, Samuels MH, Whybrow PC, Bauer M. Psychiatric and Cognitive Effects of Hypothyroidism. In: Braverman LE, Cooper DS (eds.). Werner \& Ingbar's: The thyroid: a fundamental and clinical text. $10^{\text {th }} \mathrm{ed}$. Philadelphia: Lippincott Williams \& Wilkins; 2013:596-600.

5. Dujovne NV, Gazek NA, Lazzati JM, Maceiras M, et al. Variables predictivas de talla baja adulta en pacientes con hipotiroidismo adquirido grave de origen autoinmune. Arch Argent Pediatr. 2019; 117(6):388-91.

6. Chiesa A, Gruñeiro de Papendieck L, Keselman A, Heinrich JJ, et al. Final height in long-term primary hypothyroid children. J Pediatr Endocrinol Metab. 1998; 11(1):51-8.

7. Van Vliet G, Deladoey J. Hypothyroidism in infants, children, and adolescents: adquired hypothyroidism. In: Braverman LE, Cooper DS (eds.). Werner \& Ingbar`s: The thyroid: a fundamental and clinical text. $10^{\text {th }} \mathrm{ed}$. Philadelphia: Lippincott Williams \& Wilkins; 2013.Págs.797-891.

8. Vulsma T, De Viijlder JJM. Genetic defects causing hypothyroidism. In: Braverman LE, Cooper DS (eds.). Werner \& Ingbar`s: The thyroid: a fundamental and clinical text. $10^{\text {th }}$ ed. Philadelphia: Lippincott Williams \& Wilkins; 2013. Págs.535- 51.

9. Wassner AJ. Pediatric Hypothyroidism: Diagnosis and Treatment. Paediatr Drugs. 2017; 19(4):291-301.

10. García M, Fernández A, Moreno JC. Central hypothyroidism in children. Endocr Dev. 2014; 26:79-107.

11. Diaz A, Lipman Diaz EG. Hypothyroidism. Pediatr Rev. 2014; 35(8):336-47.
12. DeVries L, BulvikS, Phillip M.Chronic autoimmune thyroiditis in children and adolescents: at presentation and during long-term follow-up. Arch Dis Child. 2009; 94(1):33-7.

13. Radetti G. Clinical Aspects of Hashimoto's Thyroiditis. In: Szinnai G (ed.). Paediatric Thyroidology. Endocr Dev. Basel: Karger; 2014; 26:158-70.

14. Botero D. Tiroides en la infancia. En Pombo M, Audí L, Calzada R, Cassorla F, et al (eds.). Tratado de Endocrinología Pediátrica. $4 .{ }^{\text {ta }}$ ed. Madrid: Mc Graw-Hill; 2009.Págs.390-2.

15. Gravholt CH, Andersen NH, Conway GS, Dekkers OM, et al. Clinical practice guidelines for the care of girls and women with Turner syndrome: proceedings from the 2016 Cincinnati International Turner Syndrome Meeting. Eur J Endocrinol. 2017; 177(3):G1-70.

16. Bull MJ, Committee on Genetics. Health supervision for children with Down syndrome. Pediatrics. 2011;128(2):393406. Erratum in: Pediatrics. 2011; 128(6):1212.

17. Leung AKC, Leung AAC. Evaluation and management of the child with hypothyroidism. World J Pediatr. 2019; 15(2):124-34.

18. Rivkees S. Thyroid Disorders in Children and adolescent. In: Sperling M (ed.). Pediatric Endocrinology. 4h ed. Philadelphia: Saunders; 2014.Págs.444-52.

19. La Franchi S. Trastornos de la glándula tiroides. En: Behrman R, Jenson H Kliegman R. Nelson Tratado de Pediatría. 16a ed. Madrid: McGraw Hill; 2000; 575:1853-9.

20. Sklar C, Whitton J, Stovall M, Green D, et al. Abnormalities of the thyroid in survivors of hodgkin's disease: Data from the childhood cancer survivor study. J Clin Endocrinol Metab. 2000; 85(9):3227-32.

21. Van Wyk, Grumbach MM. Syndrome of precocious menstruation and galactorrhea in juvenile hypothyroidism: an example of hormonal overlap in pituitary feedback. $J \mathrm{Pe}$ diatr. 1960; 57(3):416-35.

22. Pringle PJ, Stanhope R, Hindmarsh P, Brook CG. Abnormal pubertal development in primary hypothyroidism. Clin Endocrinol (Oxf). 1988; 28(5):479-86.

23. Foley TP Jr, Abbassi V, Copeland KC, Draznin MB. Hypothyroidism Caused by Chronic Autoinmune Thyroiditis in Very Young Infants. N Engl J Med. 1994; 330(7):466-8.

24. Lazar L, Frumkin RB, Battat E, Lebenthal Y, et al. Natural history of thyroid function tests over 5 years in a large pediatric cohort. J Clin Endocrinol Metab. 2009; 94(5):1678-82.

25. Beck-Peccoz P, Rodari G, Giavoli C, Lania A. Central hypothyroidism- a neglected thyroid disorder. Nat Rev Endocrinol. 2017; 13(10):588-98.

26. Van Vliet G, Deladoey J. Interpreting minor variations in thyroid function or echostructure: treating patients, not numbers or images. Pediatr Clin North Am. 2015; 62(4):92942.

27. Gallizzi R, Crisafulli C, Aversa T, Salzano G, et al. Subclinical hypothyroidism in children: is it always subclinical? Ital J Pediatr. 2018; 44(1):25.

28. Capalbo D, Alfano S, Polizzi M, Di Mase R, et al. Cognitive Function in Children With Idiopathic Subclinical Hypothyroidism: Effects of 2 Years of Levothyroxine Therapy. J Clin Endocrinol Metab. 2020; 105(3):dgaa046.

29. Salerno M, Capalbo D, Cerbone M, De Luca F. Subclinical hypothyroidism in childhood - current knowledge and open issues. Nat Rev Endocrinol. 2016; 12(12):734-46.

30. Bagnoli F, Farmeschi L, Mappini S, Grosso S. Thyroid function in small forgestational age newborns: a review. J Clin Res Pediatr Endocrinol. 2013; 5(Suppl 1):2-7.

31. Liu C, Wang K, Guo J, Chen J, et al. Small for gestational age is a risk factor for thyroid dysfunction in preterm newborns. BMC Pediatr. 2020; 20(1):179.

32. Keselman A, Chiesa A, Malozowski S, Vieytes A, et al. Abnormal Responses to TRH in Children Born Small for 
S16 / Arch Argent Pediatr 2021;119(1):S8-S16 / Subcomisiones, Comités y Grupos de Trabajo

Gestational Age That Failed to Catch Up. Horm Res. 2009; 72(3):167-71.

33. Whooten R, Schmitt J, Schwartz A. Endocrine manifestations of Down syndrome. Curr Opin Endocrinol Diabetes Obes. 2018; 25(1):61-6.

34. Aversa T, Salerno M, Radetti G, Faienza MF, et al. Peculiarities of presentation and evolution over time of Hashimoto's thyroiditis in children and adolescents with Down's syndrome. Hormones (Athens). 2015; 14(3):410-6.

35. Popova G, Paterson WF, Brown A, Donaldson MDC. Hashimoto's Thyroiditis in Down's syndrome: clinical presentation and evolution. Horm Res. 2008; 70(5):278-84.

36. Amr NH. Thyroid disorders in subjects with Down syndrome: an update. Acta Biomed. 2018; 89(1):132-9.

37. Gruneiro de Papendieck L, Chiesa A, Bastida MG, Alonso
G, et al. Thyroid dysfunction and high thyroid stimulating hormone levels in children with Down's syndrome. J Pediatr Endocrinol Metab. 2002; 15(9):1543-8.

38. Iughetti L, Predieri B, Bruzzi, Pedreiri F, et al. Ten-year longitudinal study of thyroid function in children with Down's syndrome. Horm Res Paediatr. 2014; 82(2):113-21.

39. DeGroot LJ. The Non-Thyroidal Illness Syndrome. In. Feingold KR, Anawalt B, Boyce A, Chrousos G, et al (eds.). Endotext [Internet]. South Dartmouth (MA): MDText.com, Inc.; 2000.

40. Ellerbroek V, Warncke K, Köhle J, Bonfig W. A Levothyroxine dose recommendation for the treatment of children and adolescents with autoimmune thyroiditis induced hypothyroidism. J Pediatr Endocrinol Metab. 2013; 26(1112):1023-8. 